\title{
Collaborative Involvement of Indigenous Youth in the Design and Evaluation of Digital Mental Health Interventions: A Scoping Review Protocol
}

Josie Povey ( $\square$ josie.povey@menzies.edu.au )

Menzies School of Health Research, Charles Darwin University, Casuarina campus, Ellengowan Drive, Casuarina, NT, Australia https://orcid.org/0000-0001-6961-4674

\section{Buaphrao Raphiphatthana}

Menzies School of Health Research: Charles Darwin University

\section{Michelle Torok}

University of New South Wales

\section{Tricia Nagel}

Menzies School of Health Research: Charles Darwin University

\section{Fiona Shand}

University of New South Wales

\section{Michelle Sweet}

Menzies School of Health Research: Charles Darwin University

\section{Anne Lowell}

Charles Darwin University

\section{Kylie Dingwall}

Menzies School of Health Research: Charles Darwin University

\section{Protocol}

Keywords: Digital mental health, indigenous, adolescent, young adult, scoping review, participatory

Posted Date: September 14th, 2020

DOl: https://doi.org/10.21203/rs.3.rs-75519/v1

License: (c) (i) This work is licensed under a Creative Commons Attribution 4.0 International License. Read Full License 


\section{Abstract}

Background: Indigenous youth worldwide are at greater risk of developing mental health concerns due to ongoing inequity and disadvantage. Digital mental health solutions are identified as a potential approach to improving access to mental health treatment for Indigenous youth, with evidence of acceptability and effectiveness beginning to emerge. Although collaborative design, development and evaluation is widely recognised as necessary to improving the acceptability of these tools, there is limited evidence to guide engagement of Indigenous youth in these processes. The objective of this scoping review is to map evidence regarding the collaborative involvement of Indigenous youth in the design and/or evaluation of digital mental health interventions.

Methods: Scoping review methodology includes six stages, 1) identifying research question; 2) identifying relevant studies; 3 ) developing a study selection and data extraction method; 4) charting the data; 5) collating, summarising and reporting results. Additionally, Step 6) consultation, engages a male and female Indigenous health researcher in reviewing protocols, analysis and findings, enhancing credibility and ensuring findings are informed by Indigenous worldviews. Searches for relevant literature are undertaken in the following databases: EBSCOhost databases (Academic Search Premiere, Computer and Applied Science complete, CINAHL Plus with Full text, MEDLINE with full text, APA PsychArticles, Psychology and Behavioural Sciences collection, APA PsychInfo), PubMed, and Scopus. In addition, Infomit and Google (limited to the first 200 results) are searched for grey literature. All primary studies and grey literature in English that meet eligibility criteria are included. Data, including; study methods, methodologies, digital mental health program details, participant information and engagement and reporting processes are extracted and included for analysis. Data extraction variables are guided by the Consolidated Criteria for Strengthening Reporting of Health Research (CONSIDER statement). This statement provides a best practice checklist for reporting research involving Indigenous peoples. This scoping review protocol has been registered with Open Science Framework (available via osf.io/2nkc6).

Discussion: To date, there are no reviews which critically analyse engagement of Indigenous youth in the development and evaluation of youth-specific digital mental health interventions. This review will aim to fill that gap and appraise alignment of current practice with best practice guidelines to inform future research. It will highlight appropriate strategies for the engagement of youth in collaborative processes, providing guidance for health practitioners, policy makers, and researchers working in the field of Indigenous youth and digital mental health.

\section{Background}

The majority of Indigenous youth worldwide are resilient, proud of their culture and possess social capital beyond any other recent generation (1-3). Despite this, they remain at heightened risk of developing mental illness in adolescence compared to their non-Indigenous counterparts (4). We use the collective word 'Indigenous' to describe people who originate from a particular region, however, acknowledge the rich diversity and knowledge represented by this term. Despite the need, Indigenous youth worldwide are 
less likely to access mental health treatment than non-marginalised young people $(5,6)$. Barriers to accessing mental health treatment include stigma, fear, shame, intergenerational trauma, distrust of services and being unable to identify signs and symptoms of illness $(5,6)$. Furthermore, the location of populations in need are often decentralised, meaning long distances, increased costs and challenges in the delivery of services $(5,7)$. Treatment services are often non-existent, underfunded or occur in a localised or prescribed manner within Indigenous communities, limiting their ability to affect meaningful sustained change. Considerations of language, diversity and worldview differences are sometimes overlooked, resulting in programs that are less meaningful or acceptable to the young people they are intended to serve $(8,9)$. Despite the need for culturally safe, effective, early intervention treatments, there remains relatively few approaches which are evidence based (10). Recent increased availability of technology and connectivity has been identified as an opportunity to increase access to health services within underserved communities and marginalised youth populations (6).

Digital mental health is identified as "mental health, suicide prevention and alcohol and other drugs services delivered via a digital platform" (11). There remains limited cultural diversity in the digital mental health field, despite recognition of their potential to increase access to treatment for Indigenous populations (12-14). To date, five systematic or scoping reviews have been conducted in the area of health technologies for Indigenous populations (15-19), however only one has focused on youth and mental health interventions (19). One other related article contains a literature review and a case study (20). There is a consensus among the authors of these reviews that meaningful engagement of end users in design, development or evaluation is a necessary component of digital health solutions (15, 17-19). However, none examine in-depth the strategies undertaken to engage Indigenous youth in the design or evaluation process.

Despite the importance of end user involvement in the design and evaluation of digital mental health interventions being widely identified $(11,21,22)$, there remains a lack of clear reporting on the methods and processes undertaken $(23,24)$. A popular methodology outlined in the literature is Participatory Design (PD), which is the involvement of end users in the co-creation and evaluation of digital health resources, as partners (25). This process allows an iterative approach whereby digital resource design is continually reviewed and updated (22), a necessary process which is identified in National Guidelines for safety of digital mental health solutions (11).

Furthermore, ethical guidelines on the conduct of research with Indigenous communities emphasise the importance of cultural considerations when engaging Indigenous youth in research practices (26-29). Engagement of Indigenous youth potentially protects young people and communities from being detrimentally affected and disempowered and allows better opportunity for self-determination $(26,30$, 31). A review of ethical standards in Indigenous research, has led to specific guidelines for reporting, such as the Consolidated Criteria for Strengthening Reporting of Health Research involving Indigenous peoples (CONSIDER statement), which aims to improve the quality of research practices involving Indigenous peoples (32). Within this statement, researchers are provided a comprehensive checklist of recommendations which include considerations on the governance, prioritisation, relationships, 
methodologies, participation, capacity building, analysis and interpretation and dissemination, of research involving Indigenous peoples. Critically reviewing research practices ensures they uphold ethical guidelines, and safeguards and informs best practice into the future (30).

International research has identified significant strengths unique to Indigenous young people which help build and maintain resilience $(33,34)$. One such strength is a particular affinity for creative technological innovation and design $(15,35,36)$, and willingness to embrace empowerment and self-determination (1). The involvement of Indigenous young people in solutions to address their health needs is therefore as an essential component of health service design and delivery (37). For these reasons it is essential to focus effort on engagement of Indigenous youth in the design and evaluation of digital mental health solutions, to receive the most benefit. This review aims to map evidence regarding the collaborative involvement Indigenous youth in the design and evaluation of digital mental health interventions. Findings will provide recommendations for future projects aiming to collaboratively develop or evaluate digital mental health interventions with Indigenous youth.

\section{Methods}

\section{Study Design}

Scoping reviews are particularly useful in providing an overview of research on a given topic where evidence is emerging (38) and to review research processes on a given topic (39). For these reasons a scoping review was considered the most appropriate methodology. This scoping review is based on the guidelines proposed by Arksey and O'Malley (40) and the subsequent modifications proposed by Levac et al. (38) and Peters et al. (41). It involves a six-stage process which includes: 1) identifying research question; 2 ) identifying relevant studies; 3 ) developing a study selection and data extraction method, which is refined using an iterative process (38); 4) charting the data; 5) collating, summarising and reporting results. Additionally, Step 6) consultation, engages a male and female senior Indigenous researcher throughout scoping review processes, a minimum of three times, ensuring analysis and findings are informed by Indigenous worldviews. Given the iterative nature of a scoping review, changes to the protocol can be expected (41), and any changes are detailed and justified in the final reporting.

\section{Protocol}

The protocol was drafted using the Preferred Reporting Items for Systematic Reviews and Meta-Analyses Protocols (PRISMA-P) statement (see supplementary file 1). This scoping review protocol has been registered with Open Science Framework (available via osf.io/2nkc6), as scoping review protocols are not currently accepted on PROSPERO.

\section{Research question}


The objective of this review is to map evidence regarding the collaborative involvement of Indigenous youth in the design and/or evaluation of digital mental health interventions. More specifically it aims to determine:

- Which strategies, processes and methods are used to include Indigenous youth in the design or evaluation of digital mental health interventions?

- Did strategies, processes or methods used to engage Indigenous youth in design or evaluation of digital mental health interventions align with best practice guidelines?

\section{Eligibility Criteria}

\section{Type of studies}

All study designs are included. Reviews and opinion pieces are used for reference searching only and not included in analysis. If full text articles are not available, corresponding authors are contacted. The emergence of the internet in the mid 1990's provided opportunities for health professionals to explore alternatives to face to face care (42), and literature on tele-psychiatry appeared not long after (43). Therefore, studies reported from January $1^{\text {st }} 1990$ to August $31^{\text {st }} 2020$ are eligible. Only studies reported in English are included due to limitations on time and resources.

\section{Participants}

Studies involving Indigenous youth, originating from Australia (Aboriginal and Torres Strait Islander), New Zealand (Maori), Canada (Inuit, First Nations people) and the United States of America (First Nations people) are eligible for inclusion. Indigenous people in these developed first world countries have experiences of colonisation, persistent health inequities and predominantly remote and rural residence. In addition, Indigenous people's health worldviews, language and cultural needs differ from mainstream populations. Youth for the purposes of this review, refer to those who are aged 10-24 years, representative of a broader definition of adolescence, as described by Sawyer et al. (44).

\section{Intervention}

Digital mental health interventions 'provide treatment and support to people with mental health disorders through telephone, mobile phone, computer and online applications, and can range from the provision of information, peer support services, virtual applications and games, through to real time interaction with trained clinicians' p.7 (45). Studies focused on developing or evaluating all digital mental health interventions are eligible. Electronic health or medical records, decision support tools for clinicians, analytic services, services that primarily provide support and education to health professionals, clinical practice management software, and clinical workflow and communication software are excluded (11). 


\section{Outcomes}

There are two broad categories of outcomes that are of interest, 1) study methodology and methods and 2) processes which increase engagement, governance, prioritisation, relationships, participation, capacity, analysis and interpretation and dissemination, as per the CONSIDER statement described earlier (32).

A full list of inclusion and exclusion criteria is included in Table 2.

Table 2: Inclusion Criteria

Inclusion Criteria

- Studies where at least $50 \%$ of participants are identified as Indigenous, or studies where Indigenous data is identified separately

- Studies where at least $50 \%$ of participants are aged $10-24$, or where data is identified separately for age

- Indigenous young people of Australia, Canada, New Zealand, United States of America

- The use of Information Communication Technology (smartphone, telephone, iPad, websites, computers and other digital devices) to deliver interventions targeting mental health (can be used for health promotion/psycho-education, prevention/early intervention, crisis intervention/suicide prevention, treatment, recovery and mutual/peer support)

- Primary focus of the study is mental health problems and/or wellbeing outcomes, including suicidality, substance use, and smoking.

Exclusion Criteria

- Not related to mental health/wellbeing (i.e., physical health needs as outcomes)

- Non-Indigenous population or Indigenous population but not from identified countries

- Young people are not involved in design or evaluation or are not the intended target audience of the resource

- Non-English language due to limitations in time/resources

- Electronic health or medical records, decision support tools for clinicians, analytic services, services that primarily provide support and education to health professionals, clinical practice management software, and clinical workflow and communication software

\section{Search methods and identification of studies}

\section{Search Strategy}


Following recommendations by Joanna Briggs Institute (46) a three step search process is used. First, two independent reviewers (JP, BR) undertake a limited search of the databases (EBSCOhost and PubMed). Search terms and pilot database search results are shown in supplementary file 2. Titles, abstracts and keywords of retrieved articles are reviewed to find additional search terms, before three reviewers (JP, BR, MT) meet to finalise keywords. Updated search terms are then used by two independent reviewers (JP, BR) to conduct a second search across all databases, including those aimed at identifying grey literature. Databases include; EBSCOhost databases (Academic Search Premiere, Computer and Applied Science complete, CINAHL Plus with Full text, MEDLINE with full text, APA PsychArticles, Psychology and Behavioural sciences collection, APA PsychInfo); PubMed; Scopus; Informit and Google (limited to the first 200 results). Lastly, reference lists of potential studies and reviews are examined for additional studies. If full text is not available, corresponding authors are contacted.

\section{Study selection}

The search is performed by two reviewers (JP, BR), who independently review article titles and abstracts and apply inclusion and exclusion criteria outlined in Table 2. If a study's eligibility is unclear, reviewers review full text and apply inclusion and exclusion criteria. Reviewers meet to discuss abstract screening and selection of articles. A good inter-rating agreement kappa is established at the title/abstract review stage, with two reviewers reviewing a minimum random sample of 25 studies, before proceeding to full text review. Once agreement is reached, full text articles are retrieved, and reviewed by two reviewers independently, again assessing eligibility. A final list of included articles is prepared by each reviewer. If consensus is not reached throughout each stage (abstract screening/full text review) of study selection, a third reviewer (MT) reviews the articles in question. Notes are taken describing decisions for inclusion or exclusion.

\section{Data extraction}

Two independent reviewers (JP, BR) extract relevant data. If data is missing upon review of full text, corresponding authors are contacted. Data extraction variables, outlined in Table 4, are converted into simple tables prior to data extraction. Data extraction forms are independently tested by two reviewers (JP, BR) on a random sample of five studies to ensure accuracy, consistency and validity of captured information $(38,46)$.

Table 4: Data Extraction Variables 
Variable

Study details

Description of the digital mental health resource

\section{Description or example}

Authors, date, title, journal, volume, issue, pages, country of origin, aim/objective of study

Purpose, technology type, target population, service type, therapeutic basis, mode of delivery

\section{Variables relating to processes undertaken}

Stage of development or evaluation

Methodology used

Participant demographics

Advisory boards

Data collection e.g. predesign (formative), early design, post first prototype, feasibility, efficacy or effectiveness trial, community implementation

e.g. participatory design, phenomenology, co-design, pilot study, randomised controlled trail

e.g. age, gender, ethnicity, languages spoken, English proficiency, diagnosis, role (i.e. student, patient, carer, health professional type)

e.g. leadership team, research group, consumer group

Number and duration of design or evaluation sessions, sample size, sites of data collection (e.g. School, community service), methods used (e.g. focus groups, workshops, interviews), support personnel included in design or evaluation processes (e.g interpreters, support staff)

\section{Variables relating to research best practice}

Justification for project and source

Participant feedback on design or evaluation processes

Author reflections on design or evaluation processes

Research team experience in health research

Training or support provided to Indigenous research participants

Adaptions in processes in consideration of the physical, social, economic and cultural environment of participants

Ethics board clearances

Partnerships with Indigenous corporations or communities e.g. literature, previous formative study or pilot

e.g. exit interview data, or rating scales of acceptability

Reported results, strengths, limitations and recommendations

Qualifications, time, reported relationships, credibility

e.g. provision of tablet devices to test products, training in suicide prevention to aid design processes

e.g. ethics amendments to include community consent processes, adaptions to include interpreter following initial visit or recommendation e.g. Indigenous health research ethics committees

e.g. Memorandum of Understandings, negotiation processes, agreements reached, approvals or agreements with location specific health or governance boards 
Rationale of methods/methodologies used e.g. literature, previous research, alignment with Indigenous worldviews
Consent processes reported

Dissemination practices

Indigenous involvement in stages of research

Reporting and analysis which considers the physical, social, economic and cultural environment of the participants e.g. individual, parent or collective consent, online or face to face, parties involved in consenting process (interpreter, support person)

To whom, when, platforms used

e.g. study design, funding, implementation, analysis, dissemination

e.g. consideration of social determinants of health, strengths-based reporting

\section{Collating, summarising and reporting results}

Relevant references are exported into Endnote X9, including full text. Endnote X9 allows reviewers to collaboratively manage duplicates, group and code references and add annotations and notes. General inductive analysis is used to determine themes from the data (47). Themes are independently generated and categorised by two reviewers (JP, BR). Revision and refinement of themes occurs within the research team through a series of meetings.

\section{Consultation}

Emerging themes and preliminary findings are documented and used to create discussion with two Senior Indigenous Research Officers, on at least three occasions. Preliminary findings prompt discussion and written notes are taken during consultation meetings (38). Consultation findings are presented in final reporting. This consultation phase, originally outlined by Arksey and O'Malley (40) and later refined and defined as necessary, by Levac et al. (38) enhances rigour and provides additional sources of information, perspectives, meaning, and increases the applicability of research findings. Discussion between consultants and the research team refines themes, aiming to reach consensus on results and findings.

\section{Discussion}

This scoping review aims to provide a comprehensive overview of current practices and strategies described in the literature regarding the collaborative involvement of Indigenous youth in development or evaluation of digital mental health interventions. Researchers, clinicians and technology developers will benefit from these findings as they assist in establishing best practice processes for engagement of 
Indigenous youth in digital mental health approaches as well as health research more broadly. As no other review has previously examined processes of development or evaluation in depth, this review aims to assist researchers to determine the 'how' and develop clear methodology for respectful and culturally safe engagement with Indigenous youth.

\section{List Of Abbreviations}

CONSIDER Consolidated criteria for strengthening reporting of health research involving Indigenous peoples

PD Participatory design

\section{Declarations}

Ethics approval and consent to participate

Not applicable.

\section{Consent for publication}

Not applicable.

\section{Availability of data and materials}

The materials supporting the article is included as Additional files 1 and 2.

\section{Competing interests}

The authors declare no competing interests.

\section{Funding}

JP was supported by Charles Darwin University, Research Training Program Stipend Scholarship and an Ian Scott Mental Health, Australian Rotary Health Scholarship. Funders were not involved in developing the protocol.

\section{Authors' contributions}

JP designed and wrote the manuscript. BR, KD \& MT helped design and edit the manuscript. TN, AL, MS \& FS provided advice, reviewed and revised the manuscript. All authors read and approved the final manuscript.

\section{Authors information}


JP, BR, MS, KD, TN work at Menzies School of Health Research. FS, MT work for Black Dog Institute \& University of New South Wales. AL works at Charles Darwin University. Collectively the research team have extensive experience in Indigenous Health Research, digital mental health and qualitative research methods.

\section{References}

1. Azzopardi P, Blow N, Purcell T, Brown N, Ritchie T, Brown A. Investing in the health of Aboriginal and Torres Strait Islander adolescents: a foundation for achieving health equity. Medical Journal of Australia. 2020;212(5):202-4.e1.

2. Priest N, Thompson L, Mackean T, Baker A, Waters E. 'Yarning up with Koori kids' - hearing the voices of Australian urban Indigenous children about their health and well-being. Ethnicity \& health. 2017;22(6):631-47.

3. MacDonald JP, Ford JD, Willox AC, Ross NA. A review of protective factors and causal mechanisms that enhance the mental health of Indigenous Circumpolar youth. International journal of circumpolar health. 2013;72(1):21775.

4. Azzopardi PS, Sawyer SM, Carlin JB, Degenhardt L, Brown N, Brown AD, et al. Health and wellbeing of Indigenous adolescents in Australia: a systematic synthesis of population data. The Lancet. 2018;391(10122):766-82.

5. Price M, Dalgleish J. Help-seeking among indigenous Australian adolescents: Exploring attitudes, behaviours and barriers. Youth Studies Australia. 2013;32(1):10-8.

6. Robards F, Kang M, Usherwood T, Sanci L. How marginalized young people access, engage with, and navigate health-care systems in the digital age: Systematic review. Journal of Adolescent Health. 2018;62(4):365-81.

7. Walker B, Stomski N, Price A, Jackson-Barrett E. Perspectives of Indigenous people in the Pilbara about the delivery of healthcare services. Australian Health Review. 2014;38(1):1.

8. Cass A, Lowell A, Christie M, Snelling P, Flack M, Marrnganyin B, et al. Sharing the true stories: Improving communication between Aboriginal patients and healthcare workers. Medical Journal of Australia. 2002;176(10):466.

9. Lowell A, Maypilama E, Yikaniwuy S, Rrapa E, Williams R, Dunn S. "Hiding the story": indigenous consumer concerns about communication related to chronic disease in one remote region of Australia. International journal of speech-language pathology. 2012;14(3):200-8.

10. Antonio M, Chung-Do J. SYSTEMATIC REVIEW OF INTERVENTIONS FOCUSING ON INDIGENOUS ADOLESCENT MENTAL HEALTH AND SUBSTANCE USE. American Indian and Alaska Native Mental Health Research (Online). 2015;22(3):36-56.

11. Australian Commission on Safety and Quality in health care. National Safety and Quality Digital Mental Health Standards: Draft. Canberra: Australian Commission on Safety and Quality in health care; 2020. 
12. Merry S. You can take a horse to water... Lessons learned and next steps in realizing the promise and addressing the challenges of digital mental health. IISRI: The next generation; 15th Febuary 2019; Auckland, New Zealand2019.

13. Orji R, Mandryk RL. Developing culturally relevant design guidelines for encouraging healthy eating behavior. International Journal of Human - Computer Studies. 2014;72(2):207.

14. Oyibo K, Orji R, Vassileva J. Developing Culturally Relevant Design Guidelines for Encouraging Physical Activity: a Social Cognitive Theory Perspective. Journal of Healthcare Informatics Research. 2018;2(4):319-52.

15. Jones L, Jacklin K, O'Connell ME. Development and Use of Health-Related Technologies in Indigenous Communities: Critical Review. J Med Internet Res. 2017;19(7):e256.

16. Brusse C, Gardner K, McAullay D, Dowden M. Social Media and Mobile Apps for Health Promotion in Australian Indigenous Populations: Scoping Review. J Med Internet Res. 2014;16(12):e280.

17. Hobson GR, Caffery LJ, Neuhaus M, Langbecker DH. Mobile Health for First Nations Populations: Systematic Review. JMIR Mhealth Uhealth. 2019;7(10):e14877.

18. Reilly R, Stephens J, Micklem J, Tufanaru C, Harfield S, Fisher I, et al. Use and uptake of web-based therapeutic interventions amongst Indigenous populations in Australia, New Zealand, the United States of America and Canada: a scoping review. Systematic Reviews. 2020;9(1):123.

19. Toombs E, Kowatch KR, Dalicandro L, McConkey S, Hopkins C, Mushquash CJ. A systematic review of electronic mental health interventions for Indigenous youth: Results and recommendations. Journal of telemedicine and telecare. 2020:1357633X19899231.

20. Hensel JM, Ellard K, Koltek M, Wilson G, Sareen J. Digital Health Solutions for Indigenous Mental Well-Being. Current Psychiatry Reports. 2019;21(8):68.

21. Orlowski SK, Lawn S, Venning A, Winsall M, Jones GM, Wyld K, et al. Participatory Research as One Piece of the Puzzle: A Systematic Review of Consumer Involvement in Design of Technology-Based Youth Mental Health and Well-Being Interventions. JMIR Human Factors. 2015;2(2):e12.

22. Thabrew H, Fleming T, Hetrick S, Merry S. Co-design of eHealth Interventions With Children and Young People. Frontiers in Psychiatry. 2018;9(481).

23. Vandekerckhove P, DeMul M, Bramer WM, DeBont AA. Generative Participatory Design Methodology to Develop Electronic Health Interventions: Systematic Literature Review. J Med Internet Res. 2020;22(4):e13780.

24. Moore G, Wilding H, Gray K, Castle D. Participatory Methods to Engage Health Service Users in the Development of Electronic Health Resources: Systematic Review. J Participat Med. 2019;11(1):e11474.

25. Hagen P, Collin P, Metcalf A, Nicholas M, Rahilly K, Swainston N. Participatory Design of evidencebased online youth mental health promotion, prevention, early intervention and treatment. Melbourne: Young and Well Cooperative Research Centre; 2012.

26. Hudson M, Milne M, Russell K, Smith B, Reynolds P, Atatoa-Carr P. The development of guidelines for indigenous research ethics in Aotearoa/New Zealand. In: Drugge A-L, editor. Ethics in Indigenous 
Research, Past Experiences - Future Challenges. Umea, Sweden: Vaartoe Centre for Sami Research, Umea University; 2016. p. 157-74.

27. Hudson M, Milne M, Reynolds F, Russell CL, Smith BB. Te Ara Tika Guidelines for Māori research ethics: A framework for researchers and ethics committee members. Health Research Council of New Zealand; 2009.

28. NHMRC. Ethical conduct in research with Aboriginal and Torres Strait Islander Peoples and communities: Guidelines for researchers and stakeholders. 2018.

29. Panel on Research Ethics. TCPS 2 (2018) - Chapter 9: Research Involving the First Nations, Inuit and Métis Peoples of Canada Cananda: Government of Canada; 2020 [Available from: https://ethics.gc.ca/eng/tcps2-eptc2_2018_chapter9-chapitre9.html.

30. Fitzpatrick EFM, Martiniuk ALC, D’Antoine H, Oscar J, Carter M, Elliott EJ. Seeking consent for research with indigenous communities: a systematic review. BMC Medical Ethics. 2016;17(1):65.

31. Maar MA, Beaudin V, Yeates K, Boesch L, Liu P, Madjedi K, et al. Wise Practices for Cultural Safety in Electronic Health Research and Clinical Trials With Indigenous People: Secondary Analysis of a Randomized Clinical Trial. J Med Internet Res. 2019;21(11):e14203.

32. Huria T, Palmer SC, Pitama S, Beckert L, Lacey C, Ewen S, et al. Consolidated criteria for strengthening reporting of health research involving indigenous peoples: the CONSIDER statement. BMC medical research methodology. 2019;19(1):173.

33. Salmon M, Doery K, Dance P, Chapman J, Gilbert R, Williams R, et al. Defining the Indefinable: Descriptors of Aboriginal and Torres Strait Islander Peoples' Cultures and their Links to Health and Wellbeing.; 2018.

34. Salmon M, Skelton F, Thurber K, Gosling J, Lovett R, Walter M. Intergenerational and early life influences on the well-being of Australian Aboriginal and Torres Strait Islander children: overview and selected findings from Footprints in Time, the Longitudinal Study of Indigenous Children. Journal of developmental origins of health and disease. 2019;10(1):17-23.

35. Kral I. Plugged in: Remote Australian Indigenous youth and digital culture. Canberrra: Australian National University; 2010.

36. Povey J, Mills PPJR, Dingwall KM, Lowell A, Singer J, Rotumah D, et al. Acceptability of Mental Health Apps for Aboriginal and Torres Strait Islander Australians: A Qualitative Study. J Med Internet Res. 2016;18(3):e65.

37. World Health Organisation. Engaging young people for health and sustainable development: strategic opportunities for the World Health Organization and partners. Geneva: World Health Organization; 2018.

38. Levac D, Colquhoun H, O'Brien KK. Scoping studies: advancing the methodology. Implementation Science. 2010;5(1):69.

39. Munn Z, Peters MDJ, Stern C, Tufanaru C, McArthur A, Aromataris E. Systematic review or scoping review? Guidance for authors when choosing between a systematic or scoping review approach. BMC medical research methodology. 2018;18(1):143. 
40. Arksey H, O'Malley L. Scoping studies: towards a methodological framework. International Journal of Social Research Methodology. 2005;8(1):19-32.

41. Peters MDJ, Godfrey CM, Khalil H, Mclnerney P, Parker D, Soares CB. Guidance for conducting systematic scoping reviews. International Journal of Evidence-Based Healthcare. 2015;13(3):141-6.

42. Barak A, Klein B, Proudfoot J. Defining internet-supported therapeutic interventions. Annals of Behaviour Medicine. 2009;8:4-17.

43. Monnier J, Knapp M, Frueh C. Recent advances in telepsychiatry: An updated review. Psychiatric Services. 2001;54(12):1604-9.

44. Sawyer S, Azzopardi P, Wickremarathne D, Patton G. The age of adolescence. The Lancet Child \& Adolescent Health. 2018;2(3):223-8.

45. Department of Health and Ageing. e-mental health strategy for Australia. In: Australia Co, editor. Canberra2012.

46. Joanna Briggs Institute. JBI reviewer's manual: Chapter 11: Scoping reviews [Available from: https://wiki.joannabriggs.org/display/MANUAL/Chapter+11\%3A+Scoping+reviews.

47. Thomas DR. A General Inductive Approach for Analyzing Qualitative Evaluation Data. American Journal of Evaluation. 2006;27(2):237-46.

\section{Supplementary Files}

This is a list of supplementary files associated with this preprint. Click to download.

- PoveyetalSupplementaryFiletwoSearchStrategy.docx

- PoveyetalSupplementaryfileonePRISMAPchecklist.docx 